\title{
Magnetic Resonance Imaging and Molecular Characterization of a Hormone-Mediated Murine Model of Prostate Enlargement and Bladder Outlet Obstruction
}

Erin M. McAuley, ${ }^{* \dagger}$ Devkumar Mustafi, ${ }^{\ddagger}$ Brian W. Simons, ${ }^{\S}$ Rebecca Valek, Marta Zamora, ${ }^{\ddagger}$ Erica Markiewicz, Sophia Lamperis, Anthony Williams, ${ }^{\varpi}$ Brian B. Roman, ${ }^{\ddagger}$ Chad Vezina, ${ }^{\dagger}$ Greg Karczmar, Aytekin Oto, and Donald J. Vander Griend

From the Committee on Molecular Pathology and Molecular Medicine, * and the Departments of Radiology ${ }^{\ddagger}$ and Surgery, ${ }^{\complement}$ Section of Urology, The University of Chicago, Chicago, Illinois; the Brady Urological Institute, ${ }^{\S}$ The Johns Hopkins School of Medicine, Baltimore, Maryland; and the Department of Comparative Biosciences, ${ }^{\dagger}$ University of Wisconsin Madison School of Veterinary Medicine, Madison, Wisconsin

Accepted for publication July 26, 2017.

Address correspondence to Donald J. Vander Griend, Ph.D., Department of Surgery, Section of Urology, The University of Chicago, $5841 \mathrm{~S}$ Maryland Ave., Ste. MC6038, Chicago, IL 60637. E-mail: prostate@uchicago.edu.

\begin{abstract}
Urinary complications resulting from benign prostatic hyperplasia and bladder outlet obstruction continue to be a serious health problem. Novel animal model systems and imaging approaches are needed to understand the mechanisms of disease initiation, and to develop novel therapies for benign prostatic hyperplasia. Long-term administration of both estradiol and testosterone in mice can result in prostatic enlargement and recapitulate several clinical components of lower urinary tract symptoms. Herein, we use longitudinal magnetic resonance imaging and histological analyses to quantify changes in prostatic volume, urethral volume, and genitourinary vascularization over time in response to estradiol-induced prostatic enlargement. Our data demonstrate significant prostatic enlargement by 12 weeks after treatment, with no detectable immune infiltration by macrophages or T- or B-cell populations. Importantly, the percentage of cell death, as measured by terminal deoxynucleotidyl transferase dUTP nick-end labeling, was significantly decreased in the prostatic epithelium of treated animals as compared to controls. We found no significant change in prostate cell proliferation in treated mice when compared to controls. These studies highlight the utility of magnetic resonance imaging to quantify changes in prostatic and urethral volumes over time. In conjunction with histological analyses, this approach has the high potential to enable mechanistic studies of initiation and progression of clinically relevant lower urinary tract symptoms. In addition, this model is tractable for investigation and testing of therapeutic interventions to ameliorate or potentially reverse prostatic enlargement. (Am J Pathol 2017, 187: 2378-2387; http://dx.doi.org/ 10.1016/j.ajpath.2017.07.014)
\end{abstract}

Supported by the Department of Surgery, Section of Urology, The University of Chicago; The University of Chicago Comprehensive Cancer Center; NIH/National Cancer Institute (NCI) grant P30CA014599 (University of Chicago Cancer Center); The Brinson Foundation; the Alvin Baum Family Fund; The Pierce Foundation; The University of Chicago Cancer Research Foundation Women's Board; NIH/NCI grant R01CA178431 (D.J.V.G.); NIH/National Institute of General Medical Sciences grant T32GM007183 (E.M.M.); a Ruth L. Kirschstein National Research Service Award (NRSA) Individual Predoctoral Fellowship [NIH/
National Institute of Diabetes and Digestive and Kidney Diseases (NIDDK) grant DK111131 (E.M.M.)]; NIH/NIDDK grant U54DK104310 sub 7660 (C.V.); and the Team Science Award from the University of Chicago Comprehensive Cancer Center. This work was conducted in the Integrated Small Animal Imaging Research Resource, supported in part by funding provided by The University of Chicago Comprehensive Cancer Center grant NIH/NCI P30CA014599, the Biological Sciences Division, and the Department of Radiology at The University of Chicago.

Disclosures: None declared. 
Benign prostatic hyperplasia (BPH), or prostatic enlargement, is the pathophysiological process by which the prostate gland undergoes a second, abnormal growth phase that worsens with age. ${ }^{1,2}$ At least $50 \%$ of men between the ages of 50 and 60 years show pathologic signs of $\mathrm{BPH}$, and by the age of 90 years, the incidence of clinical BPH increases to $90 \%{ }^{3,4}$ The human prostate is divided into three distinct histological zones: central, peripheral, and transition. ${ }^{5}$ Surrounding the urethra as it passes through the prostate, the transition zone is the site of nearly all clinically significant $\mathrm{BPH} .^{2}$ As a result of prostatic hyperplasia, patients can experience bladder outlet obstruction (BOO) and subsequent lower urinary tract symptoms (LUTS), both of which worsen with age. LUTS can include urinary retention, nocturia, weak stream, dribbling, pain, and frequent daytime urination. These symptoms represent substantial quality-of-life challenges and significant comorbidities. ${ }^{3}$ Although successful in some patients, some front-line treatments for BPH patients can result in significant financial and physiological burdens, and ultimately lose efficacy. ${ }^{6}$ As the success of these therapies can be shortlived, other factors may be involved in BPH pathogenesis. Currently, there is a paucity of animal models to test molecular and physiological hypotheses pertaining to the initiation and progression of BPH. This gap in knowledge represents a hurdle in our progress toward developing more effective therapies to treat $\mathrm{BPH}$.

It has long been hypothesized that BPH can result from aberrant stem cell activation and subsequent hyperproliferation, an embryonic reawakening. ${ }^{2,6}$ Lack of sufficient in vivo models, however, has made this hypothesis difficult to test directly. ${ }^{7,8}$ Foundational studies done in canines demonstrate that treatment with a combination of androgen- and estrogen-receptor agonists could induce prostatic hyperplasia. ${ }^{9}$ Moreover, the pathology of the hormonally induced hyperplasia was not fundamentally different from the spontaneous hyperplasia found in older beagles. ${ }^{9}$ Recently, it has been shown that long-term administration of both estradiol (E) and testosterone (T) using slow-release implants in mice can induce bladder obstruction and model key components of prostatic hyperplasia. ${ }^{10}$ Hormonal dysregulation results in urinary tract complications consistent with clinical BOO in men, including bladder enlargement and increase in bladder smooth muscle and collagen. ${ }^{11}$ Moreover, as a result of $\mathrm{T}+\mathrm{E}$ treatment, mice displayed a significant decrease in the size of the prostatic urethral lumen, increased prostate mass, and increased number of prostatic ducts associated with the prostatic urethra. ${ }^{11} \mathrm{~A}$ follow-up study by the same group established estrogen receptor $\alpha$ as a key mediator of BOO/BPH pathophysiology in this model, mimicking the hormonal milieu found in aging men. ${ }^{12}$ Adapting a method to use imaging analyses of these mice over time would allow us to efficiently quantify changes in prostate and urethra volume over time and measure changes in response to therapy.

Clinically, magnetic resonance imaging (MRI) has frequently been used to evaluate prostatic disease as it provides a relatively high spatial resolution as well as soft tissue contrast. ${ }^{13,14}$ Notably, multiparametric MRI (T2-weighted MRI, diffusion- weighted MRI, dynamic contrast-enhanced MRI, and MR spectroscopy) is capable of identifying prostate cancers with high sensitivity. ${ }^{15}$ MRI can potentially be used to estimate zonespecific prostatic volume, detect enlarged zones, and approximate stromal/glandular ratio. ${ }^{16-18}$ Herein, we investigate the utility of MRI with histological analyses to quantify changes in prostate volume and cellular composition after hormoneinduced $\mathrm{BOO}$ and prostatic enlargement. In our hands, we can recapitulate previously reported estradiol-mediated urethral thickening and bladder outlet obstruction in mice. ${ }^{10,12}$ Moreover, we find that MRI is a powerful tool with which to longitudinally monitor prostatic enlargement and genitourinary vascularization.

\section{Materials and Methods}

\section{Animals}

All animal care and use was approved by The University of Chicago (Chicago, IL) Institutional Animal Care and Use Committee (protocol 72294). The 11-week-old, postpubescent C57/BL6 males were castrated, as previously described. ${ }^{10,12,19}$ Briefly, animals were first anesthetized using ketamine/xylazine. After preparation of the surgical site, an incision was made in the scrotum. For each testicle, the testis, vas deferens, and attached testicular fat pad were pulled out of the incision and tied off. The testis, vas deferens, and fatty tissue were severed just below the surgical knot. The scrotal incision was closed with a nonabsorbable Nylon suture (Med-Vet, Mettawa, IL). After castration, silastic hormone pellets containing a blend of $11.25 \mathrm{mg}$ testosterone and $1.25 \mathrm{mg}$ estradiol (Sigma, St. Louis, MO) were surgically implanted to induce hormonal dysregulation, as previously described. ${ }^{10,12}$ Control mice were implanted with a $12.5-\mathrm{mg} /$ pellet per mouse testosterone pellet. Implants were used according to Sedelaar et al, ${ }^{20}$ whereby a $1-\mathrm{cm}$ implant maintains host testosterone levels at $5.3 \pm 0.5 \mathrm{ng} / \mathrm{mL}(18.2 \mathrm{nmol} / \mathrm{L})$, which is similar to eugonadal adult human males. Animals were age matched across conditions. Isoflurane anesthesia was used in all in vivo imaging studies of mice, all efforts were made to minimize any suffering, and the mice were humanely euthanized after the experiment for purposes of histology. Mice treated with $\mathrm{T}+\mathrm{E}$ who became moribund were sacrificed humanely and censured accordingly.

\section{Magnetic Resonance Imaging and Analysis}

A 9.4-Tesla small animal scanner (Bruker, Billerica, MA) with an 11.6-cm inner diameter and actively shielded gradient coils (maximum constant gradient strength for all axes, $230 \mathrm{mT} / \mathrm{m}$ ) was used to acquire MR images. ${ }^{21}$ The mouse was placed supine on an animal holder and inserted into a 30-mm-diameter quadrature mouse volume coil (Bruker). Multislice rapid acquisition with relaxation enhancement T2-weighted images with fat suppression were acquired with the following parameters: repetition time/echo time $_{\text {effective, }} 4000 / 20$ and 8000/40 milliseconds; field of 
view, $25.6 \times 25.6 \mathrm{~mm}$; matrix size, $256^{2}$; slice thickness, 0.5 $\mathrm{mm}$; rapid acquisition with relaxation enhancement factor, 4; and number of averages, 2 with 47 slices in the axial plane. For time-of-flight (TOF) angiography, a flow-compensated, T1-weighted sequence with a short repetition time and thin slices was used to maximize inflow effects and depict flowing blood as a bright signal. Parameters for TOF were as follows: repetition time/echo time effective, $_{10 / 3}$ milliseconds; flip angle, 60 degrees; field of view, $25.6 \times 25.6 \mathrm{~mm}$; matrix size, $256 \times 256$; slice thickness, $0.5 \mathrm{~mm}$; and number of averages, 4 with 47 slices. In-plane resolution for TOF was $100 \mu \mathrm{m}$, as in T2-weighted images. The acquisition time for each slice was 10.2 seconds, and the total acquisition time for this TOF sequence was 8 minutes.

An integrated software program, Amira version 5.6 (FEI Visualization Sciences Group, Burlington, MA), was used to measure the volumes of prostate and urethra, and to generate three-dimensional images. By orienting the in vivo MR images and the ex vivo MR and histology images in the same direction, MRI was accurately correlated with histopathology. Regions of interest (the prostate and the urethra) were labeled on each slice of each week of T2-weighted images. To measure blood volume, the five center slices of prostate were identified on the T2-weighted images acquired in weeks 10 and 12 after treatment. The corresponding TOF images were used to measure and label the blood volumes in those five center slices. Volume was calculated from the total number of pixels in each region of interest, multiplied by voxel volume $\left(0.005 \mathrm{~mm}^{3}\right)$. Volumes were calculated for all mice for each week before the mouse was sacrificed. A $U$-test was performed for statistical analysis. $P<0.05$ was considered significant.

\section{Preparation of Mouse Urogenital Glands for ex Vivo MRI and Histology}

After in vivo MRI studies, urogenital glands, including prostate, seminal vesicles, bladder, and penis, were excised from the mouse body and placed in 10\% formalin for 2 weeks for tissue fixation. Before ex vivo imaging, the tissue sample was removed from the formalin, rinsed daily with phosphatebuffered saline (Fisher Scientific, Waltam, MA) for 3 days, and then placed between two layers of pathology foam. Immediately before imaging, the tissue was washed twice with fomblin (Solvay Solexis, West Deptford, NJ) and then saturated with fresh fomblin. The sample was folded in plastic wrap and placed in the MRI coil for imaging. Fomblin is an oily, fluorinated polymer with no MRI signal that is used to keep the tissue moist while imaging to minimize susceptibility gradients. After ex vivo MRI, the sample was removed from the wrap, washed thoroughly in phosphate-buffered saline, and put in a histology cassette in ethanol. Because the ex vivo tissue and the hematoxylin and eosin-stained tissue were scanned in the same orientation, the ex vivo MR images bridged the gap between in vivo MRI and histology to facilitate accurate correlation. ${ }^{21}$ The same 9.4-T scanner was used to acquire ex vivo MR images, with the tissue being held in an eight-leg, low- pass, half-open birdcage coil (length, $3 \mathrm{~cm}$; width, $3 \mathrm{~cm}$; height, $2 \mathrm{~cm}$ ) that was built in the laboratory. Ex vivo threedimensional images were acquired with a T2-weighted sequence with fat suppression and with the following parameters: repetition time/echo time effective, $4000 / 20$ milliseconds; field of view, $25.6 \times 25.6 \times 8 \mathrm{~mm}$; matrix size, $384 \times 384 \times$ 120; isotropic resolution, $67 \mu \mathrm{m}$; and number of averages, 5 .

\section{Immunohistochemistry and Image Acquisition}

Sections (5 $\mu \mathrm{m}$ thick) were first baked and rehydrated using a series of xylene and ethanol gradients. Antigen retrieval was performed by heating slides with a steamer in $10 \mathrm{mmol} / \mathrm{L}$ citrate buffer ( $\mathrm{pH}$ 6) for 20 minutes. Sections were blocked and incubated with primary antibodies for 1 hour at room temperature. Antibodies were used as follows: p63 clone 4a4 (ATCC, Manassas, VA), Ki-67 (catalog number RM-9106S; Thermo Scientific, Waltham, MA), CD3 (catalog number Ab16669; Abcam, Cambridge, UK), F4/80 (catalog number MCA497GA; abD Serotec, Oxford, UK), CD31 (catalog number ab28364; Abcam), and B220 (catalog number 553085; BD, Franklin Lakes, NJ). After a tris-buffered saline wash, the antigen-antibody binding was detected with Envision+System (K4001, K4002, and K4003; Dako, Santa Clara, CA) and DAB+Chromogen (K3468; Dako). Tissue sections were briefly immersed in hematoxylin for counterstaining and were coverslipped. Histology and immunohistochemistry images were acquired on the Scanscope XT (Aperio, Buffalo Grove, IL). An average of 5 to 10 fields per mouse at each time point ( $n=2$ to 5 mice) were traced and subsequently analyzed. Positive nuclei were quantified using a Nuclear Stain or Positive Pixel algorithm in ImageScope version 12.1.0.5029 (Leica, Buffalo Grove, IL), optimized on control slides.

\section{Results}

\section{Estradiol-Mediated Hormonal Dysregulation Induces Prostatic Enlargement as Detected by MRI}

Post-pubescent male mice were castrated and surgically implanted with s.c. silastic implants containing testosterone alone ( $\mathrm{T}$ control) or a combination of testosterone and estradiol $(\mathrm{T}+\mathrm{E}) .{ }^{10}$ Mice from both the control and experimental arms were evaluated using MRI at 2- to 4-week intervals after implantation and then sacrificed accordingly for histological analyses (Figure 1A). Figure 1 illustrates an increase in overall prostate volume when measured longitudinally via MRI. A significant increase in prostate volume in mice treated with $\mathrm{T}+\mathrm{E}$ was observed when compared to $\mathrm{T}$ controls; this increase in volume was statistically significant at 12 weeks after hormone implantation (Figure 1, B and C). Serial analyses of prostate volume over time demonstrated that the prostatic volume of mice in the $\mathrm{T}+\mathrm{E}$ group showed approximately a twofold change in prostate volume $(P=0.01)$ as compared to the control group, whereas control mice showed no overall significant change over time (Figure 1, C and D). 

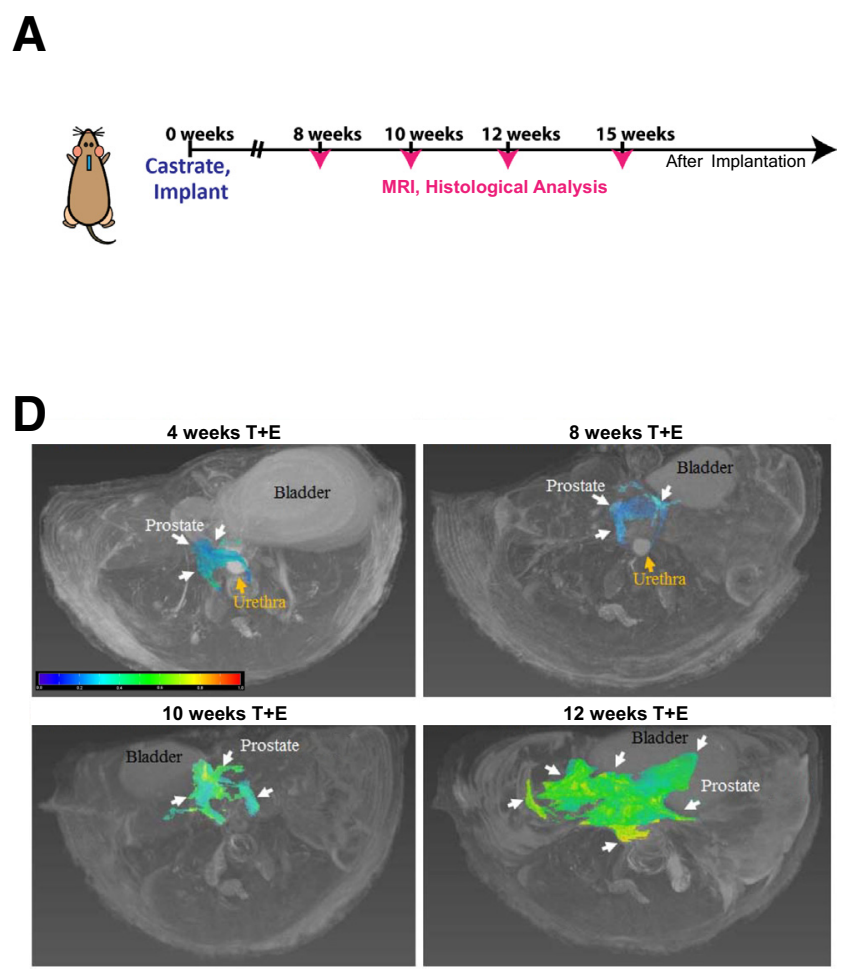

\section{Prostate Enlargement Is Associated with Decreased Urethral Volume and Increased Blood Vessel Volume}

To further characterize the prostatic enlargement associated with $\mathrm{T}+\mathrm{E}$ treatment, MRI was used to measure prostate blood volumes and prostatic urethra volume. Although some variability among mice was noted, quantification of prostatic urethral volume demonstrated a significant reduction in volume at 12 weeks after hormone treatment $(P=0.02)$ (Figure 2A). Multiple mice exhibited a distended urethra as a result of $\mathrm{T}+\mathrm{E}$ treatment (Figure 2B). Urine retention in the bladders of multiple mice after the 12week treatment time point was observed. Prostate blood volumes were measured using TOF angiography. Mice treated with $\mathrm{T}+\mathrm{E}$ had an average blood volume of 15.3 $\mathrm{mm}^{3}$, whereas the average blood volume of the T-control treated mice was $9.2 \mathrm{~mm}^{3}(P=0.01$ ) (Figure 2, C and D). This increase in blood volumes was corroborated by immunohistochemical staining of CD31, an endothelial cell marker, and was observed even at the 10 -week time point before notable prostatic enlargement (Figure 2E).

\section{Histological Analysis of Enlarged Prostates}

Ex vivo MRI was used to capture more high-resolution analyses of prostatic volume and determine whether certain prostatic lobes were disproportionally affected by the $\mathrm{T}+\mathrm{E}$ treatment (Figure 3). This figure illustrates that in vivo MRI images can be precisely correlated with ex vivo histology images. Histological analysis of enlarged prostate glands
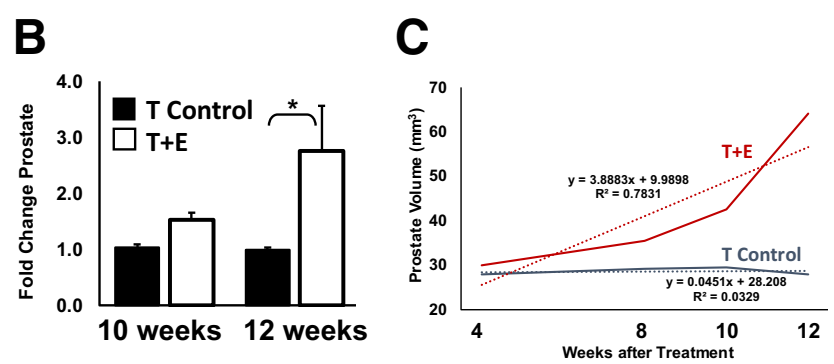

Figure 1 Longitudinal magnetic resonance imaging (MRI) analyses of prostatic enlargement in mice treated with combined testosterone $(T)$ and estradiol (E). A: Schematic of experimental approach, whereby 10-week-old male C57BL6 mice were castrated and given a s.c. silastic implant containing either testosterone alone ( $T$ control) or a 10:1 ratio of testosterone and estradiol (T+E). At 8, 10,12, and 15 weeks after castration, mice were imaged and tissues were obtained for histological analyses. B: Quantification of prostate volume by MRI demonstrates a significant increase in prostatic volume beginning at 12 weeks after treatment. C: Longitudinal analyses demonstrate an approximate doubling of prostatic volume in the $\mathrm{T}+\mathrm{E}$ condition as compared to the T-control condition. The dotted lines represent a best-fit curve for each of the cohorts. D: Representative MR images illustrating changes in prostate volume as a function of time in mice treated with $\mathrm{T}+\mathrm{E}$. The prostate volume is indicated in color-a color bar is shown for the hyperintense prostate lesion on T2-weighted images; other anatomical sites (bladder and urethra) are also noted. The heat map correlates to fluid density, where red is more intense and blue is less intense. ${ }^{*} P<0.05$.

$(\mathrm{T}+\mathrm{E})$ showed predominantly normal histology, with rare foci of epithelial hyperplasia and mild nuclear atypia in the dorsal prostate. ${ }^{22}$ Such changes are comparable to what is observed in mice at 1 to 2 years of age, even though our oldest mouse was 26 weeks at the 15 -week post-treatment time point. Stromal thickness and inflammatory cell infiltrate were similar to the T-control group and not above the background level expected in untreated mice.

\section{Prostatic Inflammatory Infiltrate Is Not Altered as a Result of Estradiol Treatment}

Given the increased blood vessel density during prostatic enlargement, we investigated whether there was an increased prostatic inflammatory infiltrate in the $\mathrm{T}+\mathrm{E}$ treated mice, and whether such an increase in immune cell infiltration might be an underpinning mechanism of prostatic enlargement. Analyses of hematoxylin and eosin sections did not reveal any overt immune cell infiltrates in the $\mathrm{T}+\mathrm{E}$ condition when compared to the T-control condition. Immunohistochemical staining and quantification of macrophages (F4/80), T cells (CD3), and B cells (B220) document no significant changes in cell populations within enlarged prostates (Figure 4). These data do not support a mechanism of inflammation-induced enlargement, but do not preclude the possibility that differential behavior of the immune cells present, or other populations, may contribute to prostatic enlargement. It is also possible that the time points assessed were not optimized for the detection of maximal immune cell infiltrate. 
Decreased Cellular Death, but Not Increased Cellular Proliferation, Contributes to Prostatic Enlargement

The observed lack of robust epithelial hyperplasia, stromal hyperplasia, or immune cell infiltrates during prostatic doubling led us to investigate whether there were significant changes in cell proliferation or death during prostatic enlargement. Tissues at 12- and 15-week time points were stained for the proliferation marker Ki-67 or using a terminal deoxynucleotidyl transferase dUTP nick-end labeling assay to detect dying cells. Quantification of staining across all prostatic lobes demonstrated no significant differences in the percentage of Ki-67-positive cells between $\mathrm{T}$-control and $\mathrm{T}+\mathrm{E}$ treated conditions (Figure $5 \mathrm{~A}$ ). In addition, we tested whether a different population of cells, such as luminal epithelial cells, was proliferating in response to $\mathrm{T}+\mathrm{E}$ treatment. We thus conducted coimmunofluorescence staining of Ki-67 along with a luminal cell-restricted cytokeratin (CK8) and a basal cell-restricted cytokeratin (CK5). Comparison of Tcontrol versus $\mathrm{T}+\mathrm{E}$ treated mice documents that $\mathrm{Ki}-67$ expression was detected in both luminal and basal epithelial cells but was not different between $\mathrm{T}$-control and $\mathrm{T}+\mathrm{E}$ conditions (Figure 5B). Detection and quantitation of cell death by terminal deoxynucleotidyl transferase dUTP nick-end labeling, however, demonstrated that the percentage of dying cells decreased dramatically in $\mathrm{T}+\mathrm{E}$ treated mice compared to $\mathrm{T}$ control mice (Figure 5C). These data demonstrate that decreased cellular death, but likely not increased cellular proliferation, in the $\mathrm{T}+\mathrm{E}$ treatment condition contributes, at least in part, to prostatic enlargement.

\section{Discussion}

Herein, we demonstrate that long-term treatment of mice with testosterone and estradiol induces a significant increase in prostatic volume with a concomitant decrease in urethral volume. We use MRI in conjunction with histological analyses to investigate the mechanistic underpinnings of progression of hormone-induced LUTS in a recently established mouse model. ${ }^{10,12}$ In vivo MRI was particularly useful in longitudinal quantification of prostatic and urethral volumes (Figures 1, B and $\mathrm{C}$, and $2 \mathrm{~A}$ ). Ex vivo MR scans provided a high-resolution image of increased prostatic cellularity after 15 weeks of hormonal treatment. Using histological analyses, we did not observe overt epithelial or stromal hyperplasia or immune infiltrate by $\mathrm{B}$ or $\mathrm{T}$ cells in prostates of $\mathrm{T}+\mathrm{E}$ treated animals as compared to controls. However, there was a significant reduction in terminal deoxynucleotidyl transferase dUTP nickend labeling-positive prostatic epithelial cells in the $\mathrm{T}+\mathrm{E}$ treated cohort at 15 weeks after treatment.

Foundational studies on the effects of steroid hormones on canine prostate growth have documented that estrogen treatment significantly augments androgen effects, inducing a fourfold increase in total canine prostate weight and DNA content. ${ }^{9,23,24}$ Furthermore, treatment of a castrated dog with estrogens in conjunction with testosterone results in either normal glandular growth or, if used in tandem with dihydrotesterone, glandular hyperplasia. Taken together, these data indicate that changing ratios of androgens and estrogens, such as in the aging process, might promote
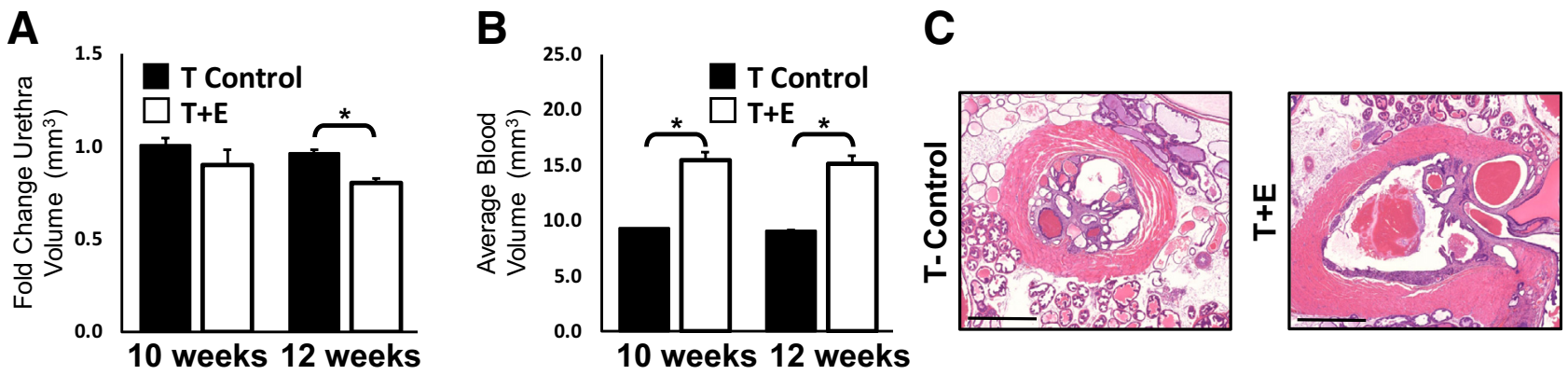

D
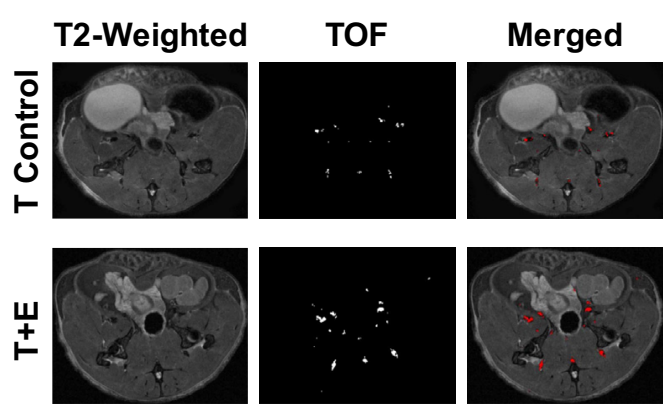

E
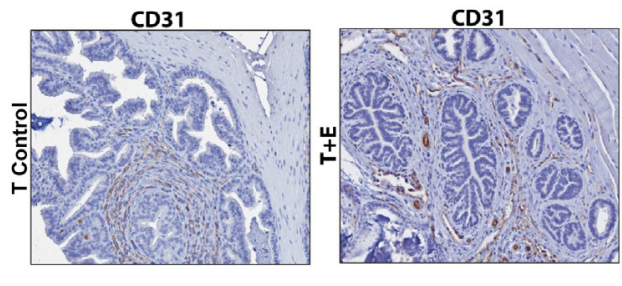

Figure 2 Urethral volume decreases and prostate blood vessel volume increases in mice with enlarged prostates. A: Quantification of prostatic urethral volume from magnetic resonance imaging demonstrates a significant decrease on prostatic enlargement. B: Quantification of prostatic blood vessel volume demonstrates a significant increase in blood vessel volume before significant prostatic enlargement at 12 weeks. C: Hematoxylin and eosin staining of representative urethral sections at 15 weeks after treatment in both the testosterone and estradiol $(\mathrm{T}+\mathrm{E})$ and $\mathrm{T}$-treated cohorts of mice reveals a narrowed urethral lumen. D: T2-weighted and time-of-flight (TOF) images illustrating changes in blood vessel volume in the T+E mice as compared to the testosterone alone (T-control) mice at 12 weeks. E: CD31 immunohistochemical staining reveals an increase in endothelial cells in prostatic glands in $\mathrm{T}+\mathrm{E}$ treated mice as compared to T controls. ${ }^{*} P<0.05$. Scale bars $=200 \mu \mathrm{m}(\mathrm{C})$. Original magnification: $\times 2(\mathrm{C}) ; \times 20(\mathrm{E})$. 


\section{T Control (15 weeks)}
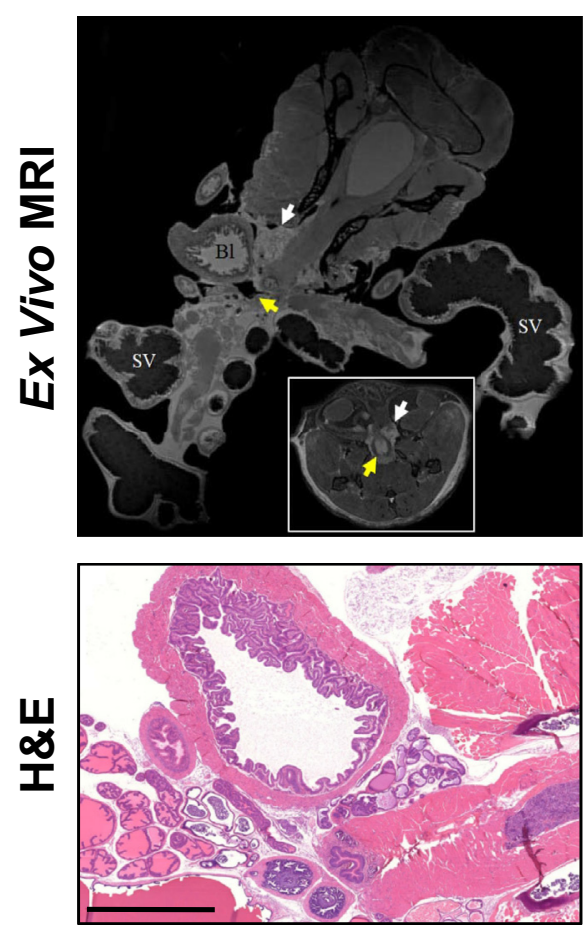

$\mathrm{T}+\mathrm{E}$ (15 weeks)
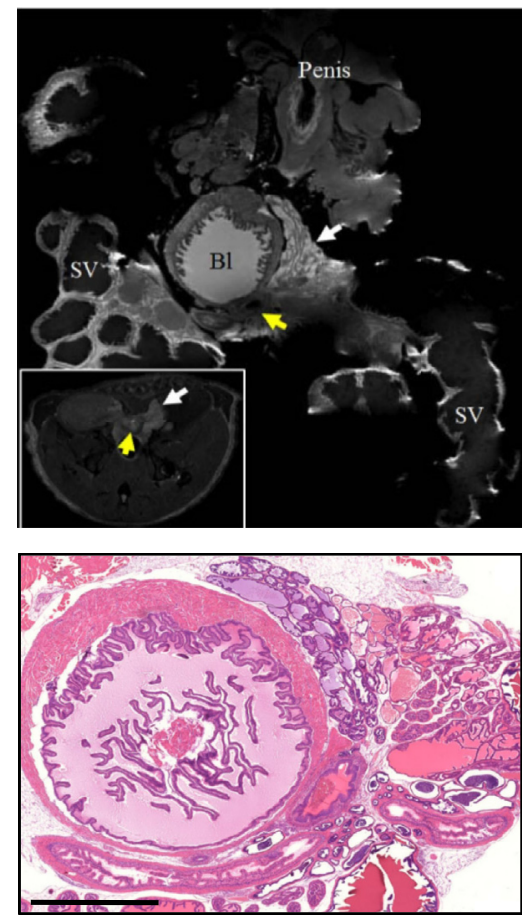

Figure 3 Ex vivo magnetic resonance imaging (MRI) and histological analyses of prostatic enlargement. Top row: High-resolution MRIs of prostates removed from the animal at the experimental end point. Insets: In vivo images of prostate before removal and ex vivo imaging. White arrows indicate the prostate, whereas the yellow arrows indicate the urethral opening. Bottom row: Hematoxylin and eosin (H\&E) staining of prostates imaged ex vivo. Scale bars $=$ $2000 \mu \mathrm{m}$ (bottom row). Original magnification: $\times 8$ (top row); $\times 1$ (insets). $\mathrm{Bl}$, bladder; SV, seminal vesicle. abnormal prostatic growth. ${ }^{9,23}$ Indeed, dogs and primates are the only mammals known to spontaneously develop symptomatic BPH. This pathophysiology has been attributed to encapsulation of the prostate by fascial layers, enabling urethral obstruction to occur as a consequence of increased prostatic tissue mass. ${ }^{10,12}$

Despite fundamental differences in prostate anatomy, the effects of hormone-mediated prostatic growth have been recapitulated in studies done using rodents. Low doses of estrogen given to prepubescent male rats sensitize the adult prostate to testosterone-mediated growth, as measured by ventral prostate wet weight. ${ }^{25}$ More recently, it was reported that long-term treatment of mice with testosterone and estradiol induces glandular prostatic growth, bladder outlet obstruction, and voiding dysfunction in male mice. ${ }^{10,12}$ Specifically, estradiol treatment of mice caused morphological changes in the proximal urethra, an increased number of periurethral prostatic ducts, and narrowing of the urethal lumen. It has been postulated that an increase in the prostate tissue encapsulated within the thick rhabdosphincter in mice may result in restriction of urinary flow, analogous to BOO found in men because of $\mathrm{BPH} .{ }^{12}$

Using MR angiography, we noted an increase in blood volumes surrounding the prostate, indicating more genitourinary vascularization as a result of estradiol treatment (Figure 2, D and E). Interestingly, microvessel density surrounding epithelial and stromal BPH nodules is enhanced relative to adjacent normal tissue and is elevated in prostates from men with symptomatic BPH, particularly those in whom surgical treatment has failed. ${ }^{26-29}$ Therefore, it may be therapeutically beneficial to reduce prostatic blood flow in men with symptomatic BPH, a notion supported by earlystage clinical studies. ${ }^{30}$ Indeed, finasteride significantly reduces prostate microvessel density in men, most potently in subepithelial stroma, where blood vessels course in close apposition to urethra urothelium. ${ }^{31-36}$ How microvessel density increases in response to the hormonal milieu found in human BPH is unclear. Studies by other laboratories indicate that new vascular development may occur via a mechanism involving hyperplasia and focal hypoxia. ${ }^{37}$

Although an increase in blood volumes suggested potential infiltration of inflammatory cells, we found no change in distribution of immune cells within the prostate as a result of $\mathrm{T}+\mathrm{E}$ treatment. The bulk ( $>90 \%)$ of immune cells found within the stroma and epithelium of human prostate are $\mathrm{T}$ lymphocytes, particularly cytotoxic $\mathrm{CD}^{+}{ }^{38}$ Typically, periglandular areas of the prostate are infiltrated by cytotoxic T lymphocytes $\left(\mathrm{CD}^{+}\right)$, whereas the fibromuscular stroma contains lymphoid aggregates composed of $50 \%$ B cells surrounded by $\mathrm{CD}^{+}{ }^{+} \mathrm{T}$ cells. In addition, macrophages are present in small populations. ${ }^{38}$ No difference in the number or distribution of the pan T-cell marker CD3 in the murine prostate was seen. Furthermore, using the macrophage marker F4/80 as well as the B-cell marker B220, we did not determine a large contribution by either population to prostatic enlargement. Conversely, human BPH tissue contains infiltrates of $\mathrm{T}$ lymphocytes, B lymphocytes, and macrophages. These cells are found to be chronically activated and coordinate cytokine release (IL-2, interferon- $\gamma$, and 
A

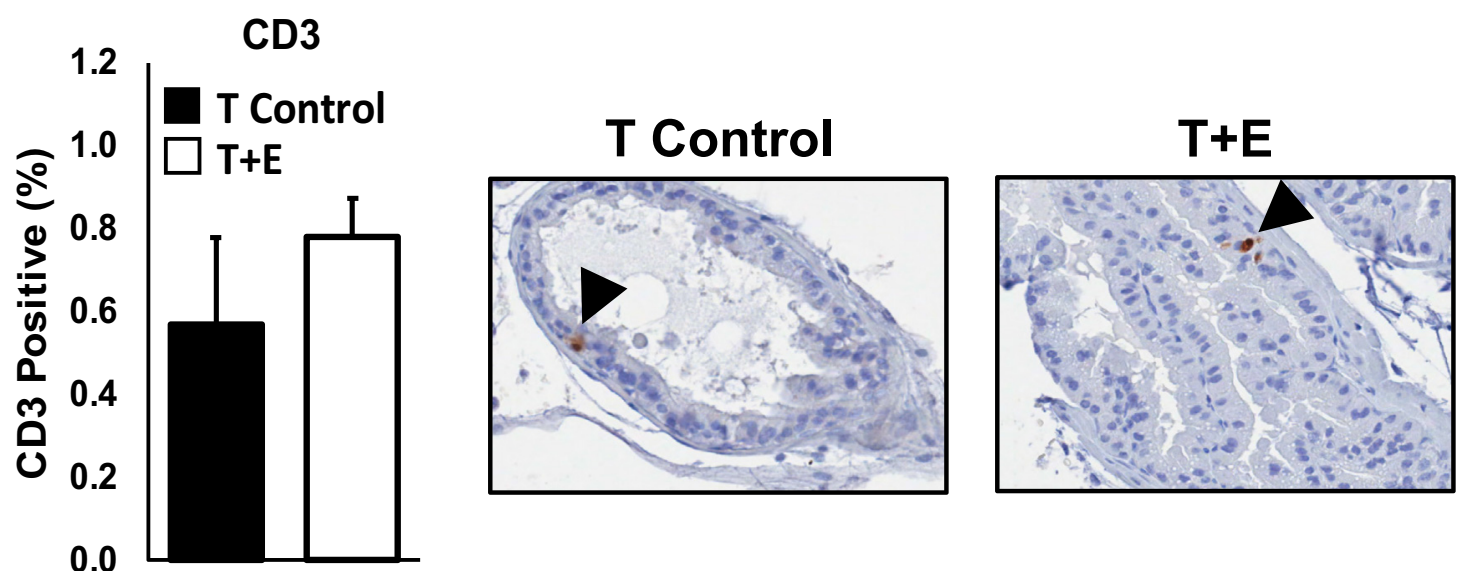

B
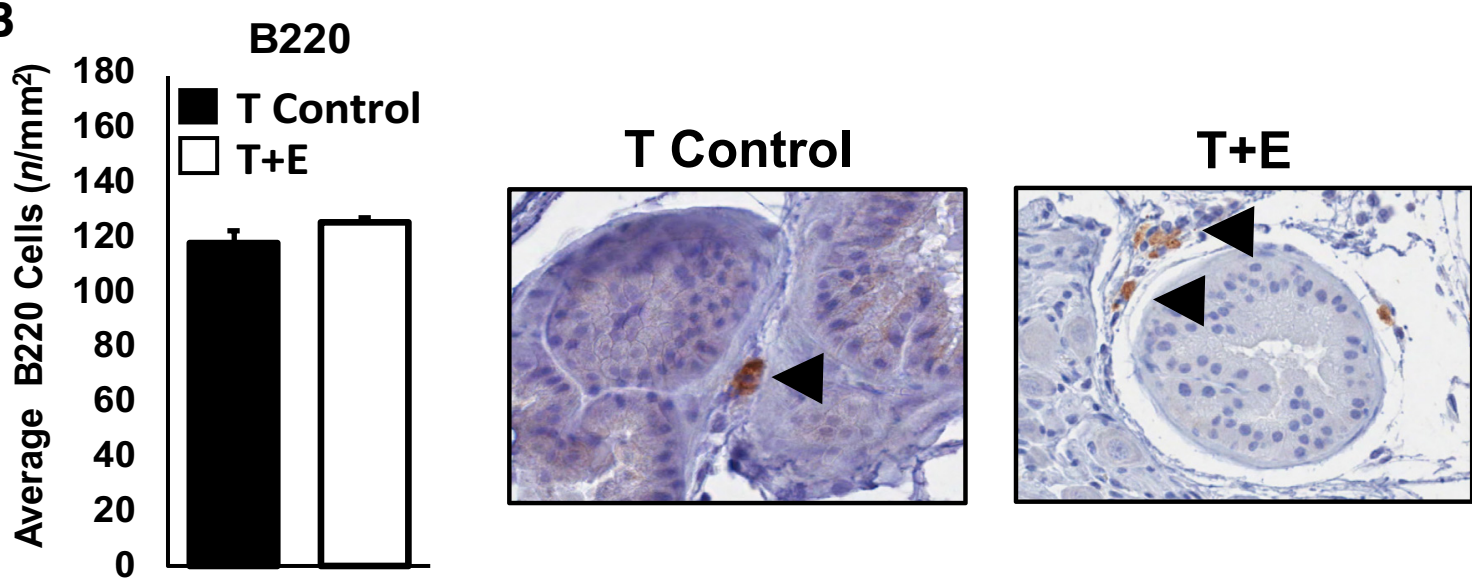

C

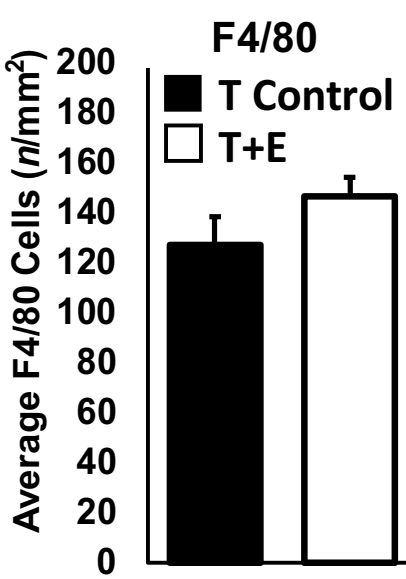

T Control

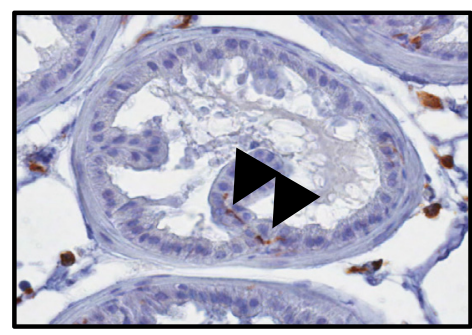

$\mathrm{T}+\mathrm{E}$

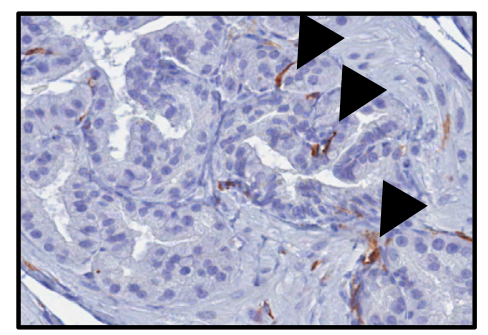

Figure 4 Quantification of immune infiltrates into enlarged versus control prostates. To measure changes in immune cell populations after prostatic enlargement, we stained and quantified the presence of T cells via CD3 (A), B cells via B220 (B), and macrophages via F4/80 (C). Data from 12- and 15-week time points, after significant prostatic enlargement, were combined for increased statistical power. We observed no statistically significant change in T-cell, B-cell, or macrophage populations on prostatic enlargement. Representative images of immunostaining are shown, each from the dorsal lobe. Arrowheads represent positive cells for immunohistochemical stain of corresponding markers. Original magnification, $\times 40(\mathbf{A}-\mathbf{C})$. $\mathrm{T}$ control, testosterone alone; $\mathrm{T}+\mathrm{E}$, testosterone and estradiol.

transforming growth factor- $\beta$ ) that may drive fibromuscular growth in $\mathrm{BPH}^{39}$ Furthermore, various proinflammatory cytokines have been discovered to be up-regulated in $\mathrm{BPH}$ tissue, particularly IL-15 in stromal cells, IL-17 in infiltrating $\mathrm{T}$ cells, interferon- $\gamma$ in basal and stromal cells, and IL-8 in epithelial cells. ${ }^{40}$ We cannot exclude the possibility that immune infiltrate occurred transiently and/or out of phase with our time points (either earlier or later in the treatment time course). Future experiments at additional time points are necessary to determine the estradiol-mediated impact of 


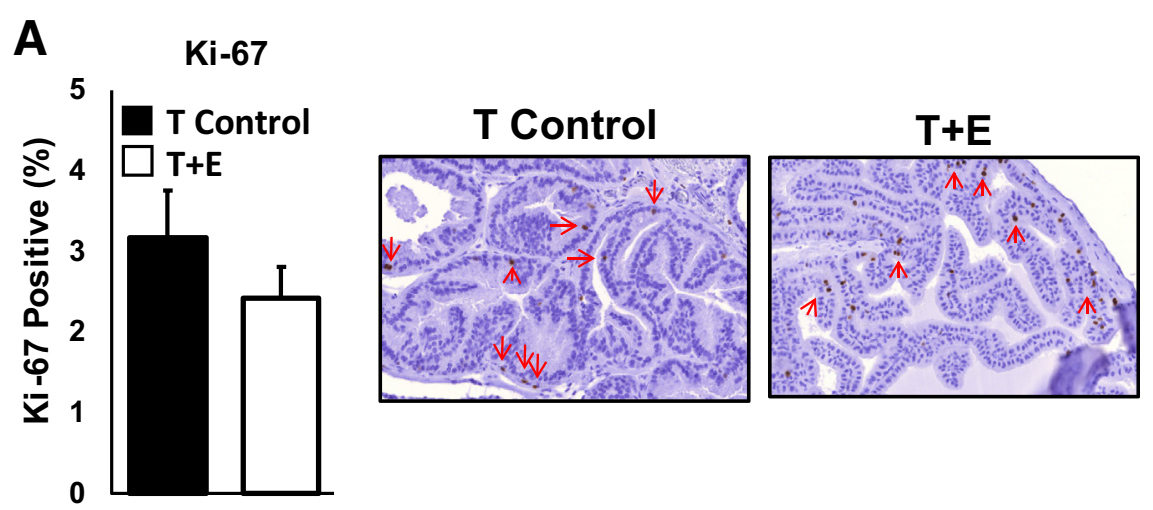

B
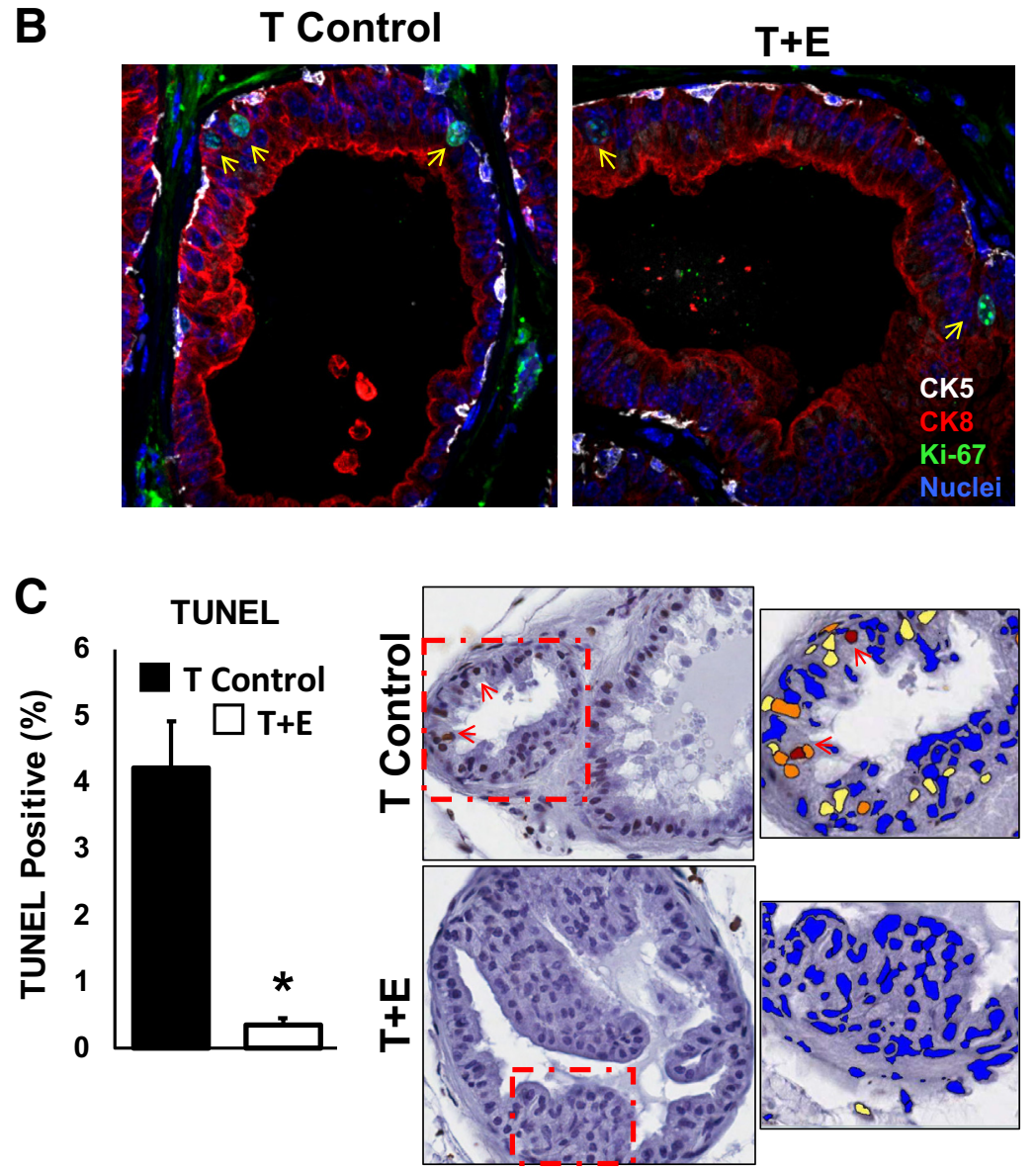

Figure 5 Decreased cell death, but not increased cell proliferation, is associated with prostatic enlargement. A: Quantification of Ki-67 positivity, used herein as an indicator of proliferation, demonstrates no significant increase in the percentage of proliferating cells in the testosterone and estradiol ( $\mathrm{T}+\mathrm{E})$ cohort of mice. Data from 12- and 15-week time points were combined for increased statistical power (5 to 10 fields quantified per mouse). Representative images of Ki-67 immunostaining (each from the dorsal lobe) are shown. Red arrows denote $\mathrm{Ki}$ $67^{+}$cells. B: Immunofluorescence analyses of Ki67 positivity (green) in both CK5-positive basal epithelial cells (white) and CK8-positive luminal epithelial cells (red) demonstrate no change in the population of epithelial cells undergoing cell proliferation in the $\mathrm{T}+\mathrm{E}$ versus testosterone alone (T-control) mice. Nuclei are counterstained with DAPI in blue. Yellow arrows denote $\mathrm{Ki}-67^{+}$cells. Representative images of immunostaining are shown. C: Quantification of cell death using terminal deoxynucleotidyl transferase dUTP nick-end labeling (TUNEL) staining via immunohistochemistry ( 5 to 10 fields quantified per mouse) demonstrates a significant reduction in cell death on prostatic enlargement. eSlide Manager software version 12.1.0.5029 (Aperio) was used to count positive cells using a Positive Nuclear Algorithm optimized on test and control slides (dotted boxes shown at higher magnification in the right panels). Representative images of immunostaining are shown. Blue cells are negative for TUNEL, yellow cells are $1+$ positivity, orange cells are $2+$ positivity, and maroon cells are $3+$ positivity (red arrows denote $3+$ TUNEL $^{+}$cell nuclei). $n=4$ mice $(\mathbf{A}) ; n=2$ mice $(\mathbf{C})$. ${ }^{\star} P<0.05$ versus $T$ control. Original magnification, $\times 40(A-C)$. various immune cell populations and cytokine signaling to LUTS pathogenesis in this model.

Interestingly, estradiol treatment of mice did not seem to significantly spur cellular hyperplasia based on Ki-67 staining. Instead, estradiol treatment was protective against cell death, as evidenced by terminal deoxynucleotidyl transferase dUTP nick-end labeling staining, coincident with volumetric changes in the prostate and urethral lumen. Longitudinal MRI revealed a significantly increased rate of prostatic enlargement at between 10 and 12 weeks of estradiol treatment based on the changing slope of the curve (Figure 1B). We speculate this may be because of the continued accumulation of actively dividing epithelial cells exceeding our detection threshold. This is not completely surprising, as historical canine studies have also reported that in established BPH the abnormal size of the aged prostate is maintained by a decrease in the rate of cell death, as opposed to an increase in the rate of cell replication. ${ }^{23}$ It is likely that the pathophysiologic response of the prostatic epithelial and stromal compartments to estradiol treatment depends on additional variables, including dosage, timing of exposure, and presence of androgens.

Overall, the hormonal dysregulation of this mouse model recapitulates several features of the clinical presentation of 
human LUTS. In accordance with the seminal publications describing this model, we observed an increase in prostatic volume, as well as a concomitant decrease in prostatic urethral volume. At the time points we evaluated, we did not observe canonical hyperplasia of prostatic stroma or epithelium, as is highly prevalent in BPH patients. Moreover, we did not observe overt immune infiltrate at experimental time points. However, we cannot exclude the possibility that immune infiltrate or epithelial proliferation occurred at other time points not evaluated via histology or MRI. Moreover, we cannot exclude the possibility of a mouse-strain-specific immune response. Future experiments are needed to more thoroughly investigate underpinning mechanisms of initiation and progression steps of LUTS in this mouse model.

This model is technically tractable as it does not require an invasive surgery and can be used in conjunction with transgenic animals. Therefore, future work can use this model to more specifically dissect the roles of relevant proteins and pathways in the development of $\mathrm{BOO}$ and BPH. Furthermore, these studies illustrate the high impact of longitudinal MRI analyses to quantify changes in prostate and urethral volume over time. Such an approach has the high potential to enable the following: i) investigations of novel therapeutic approaches to ameliorate $\mathrm{BPH}$, ii) mechanistic studies of factors contributing to either initiation or progression of $\mathrm{BPH}$, and iii) an increased understanding of the role of estrogenic hormones in regulating prostatic volume.

\section{Acknowledgments}

We thank the Section of Urology (The University of Chicago), led by Dr. Arieh Shalhav, as well as members of the Vander Griend and Szmulewitz laboratories, for helpful discussions and critiques; The University of Chicago Comprehensive Cancer Center (UCCCC), led by Dr. Michelle Le Beau, for support; the Team Science Award from the UCCCC; Linda Degenstein, for contributing the C57/BL6 mice used in this study; the Integrated Small Animal Imaging Core Facility; and the Human Tissue Resource Center core facility, led by Dr. Mark Lingen and technical director Terri $\mathrm{Li}$, as well as The University of Chicago Animal Resource Center, for expert technical assistance.

\section{References}

1. Isaacs JT: Control of Cell Proliferation and Cell Death in the Normal and Neoplastic Prostate: A Stem Cell Model. Baltimore: US Department of Health and Human Services, 1987. NIH Publication 87-2881

2. McNeal JE: Origin and evolution of benign prostatic enlargement. Invest Urol 1978, 15:340-345

3. Izumi $\mathrm{K}$, Li L, Chang $\mathrm{C}$ : Androgen receptor and immune inflammation in benign prostatic hyperplasia and prostate cancer. Clin Invest 2014 4:935-950
4. McVary KT: Clinical evaluation of benign prostatic hyperplasia. Rev Urol 2003, 5(Suppl 5):S3-S11

5. McNeal JE: The zonal anatomy of the prostate. Prostate 1981, 2:35-49

6. Isaacs JT: Prostate stem cells and benign prostatic hyperplasia. Prostate 2008, 68:1025-1034

7. Prajapati A, Gupta S, Mistry B, Gupta S: Prostate stem cells in the development of benign prostate hyperplasia and prostate cancer: emerging role and concepts. Biomed Res Int 2013, 2013:107954

8. Notara M, Ahmed A: Benign prostate hyperplasia and stem cells: a new therapeutic opportunity. Cell Biol Toxicol 2012, 28:435-442

9. DeKlerk DP, Coffey DS, Ewing LL, McDermott IR, Reiner WG, Robinson CH, Scott WW, Strandberg JD, Talalay P, Walsh PC, Wheaton LG, Zirkin BR: Comparison of spontaneous and experimentally induced canine prostatic hyperplasia. J Clin Invest 1979, 64:842-849

10. Nicholson TM, Ricke EA, Marker PC, Miano JM, Mayer RD, Timms BG, vom Saal FS, Wood RW, Ricke WA: Testosterone and 17beta-estradiol induce glandular prostatic growth, bladder outlet obstruction, and voiding dysfunction in male mice. Endocrinology 2012, 153:5556-5565

11. Nicholson TM, Sehgal PD, Drew SA, Huang W, Ricke WA: Sex steroid receptor expression and localization in benign prostatic hyperplasia varies with tissue compartment. Differentiation 2013, 85:140-149

12. Nicholson TM, Moses MA, Uchtmann KS, Keil KP, Bjorling DE, Vezina CM, Wood RW, Ricke WA: Estrogen receptor-alpha is a key mediator and therapeutic target for bladder complications of benign prostatic hyperplasia. J Urol 2015, 193:722-729

13. Guneyli S, Ward E, Thomas S, Yousuf AN, Trilisky I, Peng Y, Antic T, Oto A: Magnetic resonance imaging of benign prostatic hyperplasia. Diagn Interv Radiol 2016, 22:215-219

14. Kwak JT, Sankineni S, Xu S, Turkbey B, Choyke PL, Pinto PA, Merino M, Wood BJ: Correlation of magnetic resonance imaging with digital histopathology in prostate. Int J Comput Assist Radiol Surg 2016, 11:657-666

15. Turkbey B, Mani H, Shah V, Rastinehad AR, Bernardo M, Pohida T, Pang Y, Daar D, Benjamin C, McKinney YL, Trivedi H, Chua C, Bratslavsky G, Shih JH, Linehan WM, Merino MJ, Choyke PL, Pinto PA: Multiparametric 3T prostate magnetic resonance imaging to detect cancer: histopathological correlation using prostatectomy specimens processed in customized magnetic resonance imaging based molds. J Urol 2011, 186:1818-1824

16. Grossfeld GD, Coakley FV: Benign prostatic hyperplasia: clinical overview and value of diagnostic imaging. Radiol Clin North Am 2000, 38:31-47

17. Schiebler ML, Tomaszewski JE, Bezzi M, Pollack HM, Kressel HY, Cohen EK, Altman HG, Gefter WB, Wein AJ, Axel L: Prostatic carcinoma and benign prostatic hyperplasia: correlation of high-resolution MR and histopathologic findings. Radiology 1989, 172:131-137

18. De Visschere PJ, Vral A, Perletti G, Pattyn E, Praet M, Magri V, Villeirs GM: Multiparametric magnetic resonance imaging characteristics of normal, benign and malignant conditions in the prostate. Eur Radiol 2017, 27:2095-2109

19. Kregel S, Kiriluk KJ, Rosen AM, Cai Y, Reyes EE, Otto KB, Tom W, Paner GP, Szmulewitz RZ, Vander Griend DJ: Sox2 is an androgen receptor-repressed gene that promotes castration-resistant prostate cancer. PLoS One 2013, 8:e53701

20. Sedelaar JP, Dalrymple SS, Isaacs JT: Of mice and men-warning: intact versus castrated adult male mice as xenograft hosts are equivalent to hypogonadal versus abiraterone treated aging human males, respectively. Prostate 2013, 73:1316-1325

21. Mustafi D, Zamora M, Fan X, Markiewicz E, Mueller J, Conzen SD, Karczmar GS: MRI accurately identifies early murine mammary cancers and reliably differentiates between in situ and invasive cancer: correlation of MRI with histology. NMR Biomed 2015, 28:1078-1086

22. Shappell SB, Thomas GV, Roberts RL, Herbert R, Ittmann MM, Rubin MA, Humphrey PA, Sundberg JP, Rozengurt N, Barrios R, Ward JM, Cardiff RD: Prostate pathology of genetically engineered mice: definitions and classification: the consensus report from the Bar 
Harbor meeting of the Mouse Models of Human Cancer Consortium Prostate Pathology Committee. Cancer Res 2004, 64:2270-2305

23. Coffey DS, Walsh PC: Clinical and experimental studies of benign prostatic hyperplasia. Urol Clin North Am 1990, 17:461-475

24. Walsh PC, Wilson JD: The induction of prostatic hypertrophy in the dog with androstanediol. J Clin Invest 1976, 57:1093-1097

25. Coffey DS: Similarities of prostate and breast cancer: evolution, diet, and estrogens. Urology 2001, 57:31-38

26. Deering RE, Bigler SA, Brown M, Brawer MK: Microvascularity in benign prostatic hyperplasia. Prostate 1995, 26:111-115

27. Kojima M, Naya Y, Inoue W, Ukimura O, Watanabe M, Saitoh M, Watanabe H: The American Urological Association symptom index for benign prostatic hyperplasia as a function of age, volume and ultrasonic appearance of the prostate. J Urol 1997, 157:2160-2165

28. Foley SJ, Bailey DM: Microvessel density in prostatic hyperplasia. BJU Int 2000, 85:70-73

29. Sun QZ, Guan TY, Qi JG, Cao JY, Wu G, Yang N, Cheng ZY, Liang J, Wang Q: [Histological characteristics of the prostate in men who receive re-TURP for benign prostatic hyperplasia and their clinical significance] Chinese. Zhonghua Nan Ke Xue 2010, 16:118-122

30. Carnevale FC, Antunes AA, da Motta Leal Filho JM, de Oliveira Cerri LM, Baroni RH, Marcelino AS, Freire GC, Moreira AM, Srougi M, Cerri GG: Prostatic artery embolization as a primary treatment for benign prostatic hyperplasia: preliminary results in two patients. Cardiovasc Intervent Radiol 2010, 33:355-361

31. Marshall S, Narayan P: Treatment of prostatic bleeding: suppression of angiogenesis by androgen deprivation. J Urol 1993, 149:1553-1554

32. Donohue JF, Hayne D, Karnik U, Thomas DR, Foster MC: Randomized, placebo-controlled trial showing that finasteride reduces prostatic vascularity rapidly within 2 weeks. BJU Int 2005, 96:1319-1322
33. Lekas AG, Lazaris AC, Chrisofos M, Papatsoris AG, Lappas D, Patsouris E, Deliveliotis C: Finasteride effects on hypoxia and angiogenetic markers in benign prostatic hyperplasia. Urology 2006, 68: 436-441

34. Sutton MT, Yingling M, Vyas A, Atiemo H, Borkowski A, Jacobs SC, Kyprianou N: Finasteride targets prostate vascularity by inducing apoptosis and inhibiting cell adhesion of benign and malignant prostate cells. Prostate 2006, 66:1194-1202

35. Hochberg DA, Basillote JB, Armenakas NA, Vasovic L, Shevchuk M, Pareek G, Fracchia JA: Decreased suburethral prostatic microvessel density in finasteride treated prostates: a possible mechanism for reduced bleeding in benign prostatic hyperplasia. J Urol 2002, 167:1731-1733

36. Pareek G, Shevchuk M, Armenakas NA, Vasjovic L, Hochberg DA, Basillote JB, Fracchia JA: The effect of finasteride on the expression of vascular endothelial growth factor and microvessel density: a possible mechanism for decreased prostatic bleeding in treated patients. J Urol 2003, 169:20-23

37. Sampson N, Madersbacher S, Berger P: [Pathophysiology and therapy of benign prostatic hyperplasia] German. Wien Klin Wochenschr 2008, 120:390-401

38. De Nunzio C, Presicce F, Tubaro A: Inflammatory mediators in the development and progression of benign prostatic hyperplasia. Nat Rev Urol 2016, 13:613-626

39. Kramer G, Steiner GE, Handisurya A, Stix U, Haitel A, Knerer B, Gessl A, Lee C, Marberger M: Increased expression of lymphocytederived cytokines in benign hyperplastic prostate tissue, identification of the producing cell types, and effect of differentially expressed cytokines on stromal cell proliferation. Prostate 2002, 52:43-58

40. Briganti A: Oestrogens and prostate cancer: novel concepts about an old issue. Eur Urol 2009, 55:543-545 